\title{
Radiofrequency Ablation Versus High-Frequency Ablation In-Vivo Comparison
}

\author{
Rouane A., ${ }^{1, *}$, Bru P. ${ }^{2}$, Kourtiche D. ${ }^{1}$ \\ ${ }^{1}$ Université de Lorraine ; LIEN ; EA3440 ; 54000 ; France \\ ${ }^{2}$ Hôpital Saint Louis, service cardiologie, La Rochelle, 17019, BP 505, France
}

\begin{abstract}
The high frequency (HF) intracardiac ablation has shown its ability to obtain atrioventricular blocks (AVB) with relatively low power. In this paper, the equipment used in High Frequency (HF) ablation technique is described. The frequency used is from $500 \mathrm{kHz}$ to $1 \mathrm{MHz}$ in the case of Radiofrequency (RF) technique and $27.12 \mathrm{MHz}$ for $\mathrm{HF}$ technique. Ablation under the same conditions using the Nakagawa protocol was done. Quantitative comparison of the two methods shows efficiency of the HF technique. The results obtained in this study show that the HF technique produces lesions wider and deeper than that obtained with RF technique. Thus, HF technique is more appropriate for cardiac ablations in the ventricle. The results also showed that HF technique provides linear lesions thus delimiting the necrotic tissue from healthy tissue. With the RF technical, dimensions of the obtained lesion are $(9 \times 7 \times 5) \mathrm{mm}^{3}$ for 270 joules applied power. With the same energy, the dimensions of lesion are $(10 \times 9 \times 5) \mathrm{mm}^{3}$ for the HF Technique.
\end{abstract}

Keywords Intracardiac Ablation; High Frequency Technique, Experiments In Vivo

\section{Introduction}

For the treatment of rhythm, conduction or defects disorders, intracardiac ablation catheter is necessary to destroy diseased cells and thus replace the natural electrical pulse by a pulse delivered by a pacemaker. The frequency used is from $500 \mathrm{kHz}$ to $1 \mathrm{MHz}$ in the case of Radiofrequency (RF). $\mathrm{RF}$ ablation is often used in the treatment of various cardiac arrhythmias[1, 11]. It has often been compared to other techniques[12]. This technique is interesting in the treatment of supraventricular tachycardia. It is however limited by the rise in impedance, resulting in the formation of coagulum. Therefore, only small lesions are obtained. The need for larger wider lesions in the ventricle led us to develop an ablation technique high frequency (HF)[13, 16]. A series of experiments on ten sheeps $[14,16]$ showed the ability of HF ablation to obtain atrioventricular blocks (AVB) with relatively low power. The method that we have developed is at the validation stage.

\section{Material and Method}

\subsection{Material}

The impedance automatic adaptation is a solution to the

* Corresponding author:

Amar.rouane@lien.uhp-nancy.fr (Rouane A)

Published online at http://journal.sapub.org/ajbe

Copyright (C) 2012 Scientific \& Academic Publishing. All Rights Reserved problem faced by the RF method for the rise of impedance on the one hand and weakness, due to weak energy deposition, of the lesions obtained in the ventricle. We incorporated, between the amplifier and the load, a matching network, low losses, to optimize the energy transfer between the generator and load, which increases the efficiency of HF ablation method. The entire ablation system is showed in figure (1). We opted for a $\pi$ network with two air variable capacitors and a fixed coil. The $\pi \mathrm{LC}$ system, figure (2), comprises an L coil calculated at the working frequency, and two air capacitors $\mathrm{C} 1$ and $\mathrm{C} 2$, which vary in a range of values for the monitoring of the evolution of the load during the ablation session.

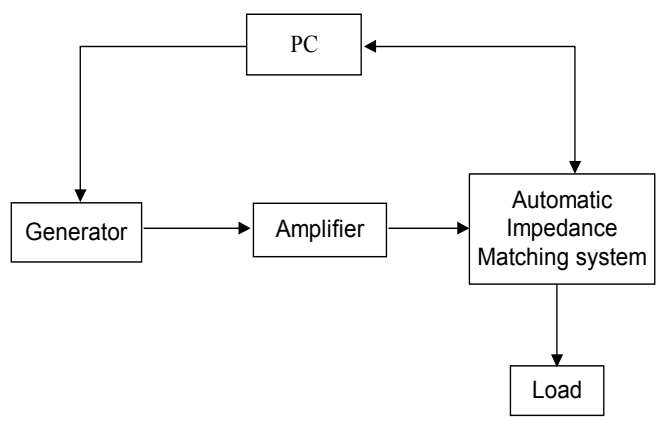

Figure 1. HF ablation system

In our case, the load is modelled schematically by a resistor in parallel with a capacitor. The matching network's role is to reduce the impedance of the load to a value corresponding to that output of the generator $(50 \mathrm{ohms})$. It is constituted by variable components (capacitors) for main- 
taining a constant value during the treatment. This phenomenon is characterised by absence of source reflected energy. It is constituted of variable elements (capacitors) to maintain this value constant during the treatment. We shall say then that there is "agreement" between the load and generator. This phenomenon is characterized by the absence of reflected energy.

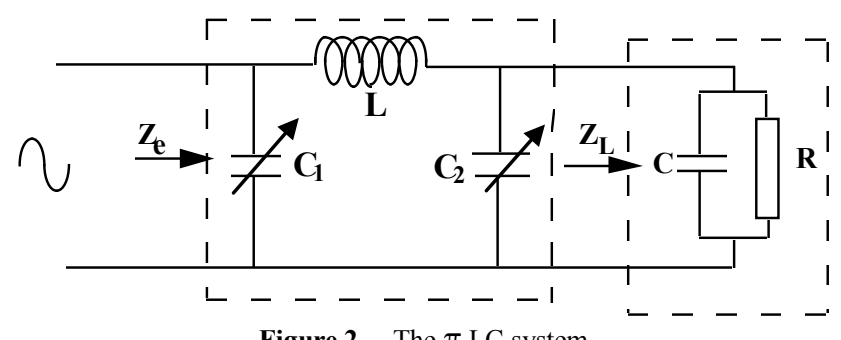

Figure 2. The $\pi \mathrm{LC}$ system

\subsection{Method}

A pig weighing $38 \mathrm{~kg}$ was used. The protocol[17] is conformable with French and institutional regulations for invasive studies on animal. An Intravenous injection of sodium pentobarbital (10 to $30 \mathrm{mg} / \mathrm{kg}$ of animal's weight) is applied before intubation. The general anaesthesia was maintained with 1.0 and up to $1.5 \%$ halothane, ketamine hydrochloride, and the assisted ventilation was used. Blood was drawn on the same day both before and at the end of the procedure in order to measure creatine kinase (CK-MB), lactic dehydrogenase, blood count, and to determine standard biochemical blood levels.
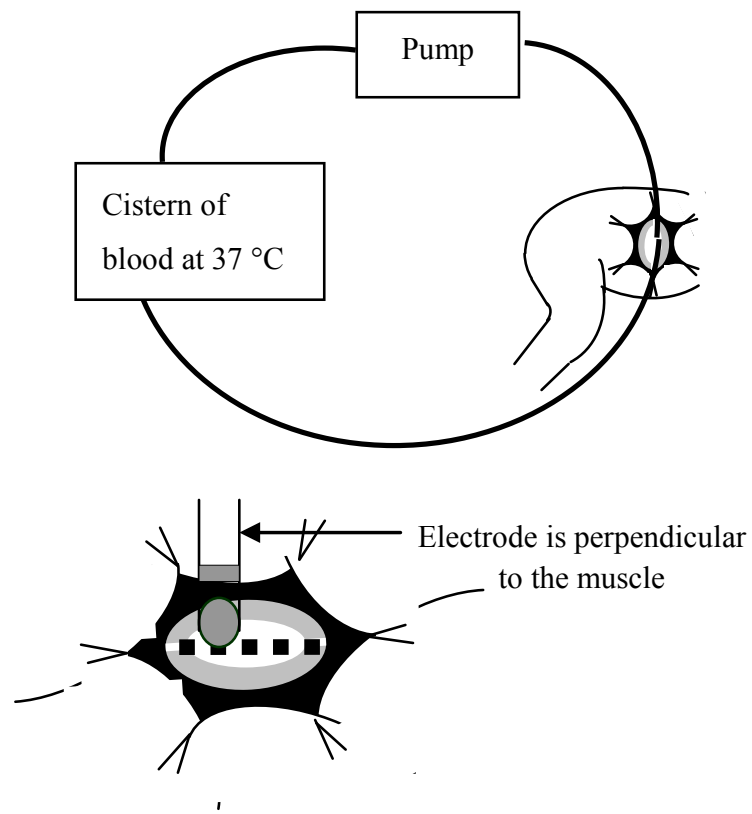

Figure 3. heparined blood and position of bipolar electrode

A standard Electrocardiogram (EKG) was displayed during the entire duration of the study. An incision of $15 \mathrm{~cm}$ long was practiced in the muscle of the thigh. We proceeded to the exposure of the surface of the muscle the thicker, keeping only the finest fascia. The edges of the incision were raised to form a crater that is filled with heparinised blood heated between $36^{\circ} \mathrm{C}$ and $37^{\circ} \mathrm{C}$, renewed at a rate of $20 \mathrm{cc}$ per minute. The lesions were created either by a probe type commonly used in RF type $7 \mathrm{~F}$ probe; either by bipolar ablation probes $27.12 \mathrm{MHz}[14,15]$. The electrode is placed perpendicularly to the thigh muscle and the catheter pression was kept constant, Figure 3.

The incision of the first sequence had to be closed before starting the contralateral thigh. The energy used varied from 5 to 50 watts and 5 to 30 seconds to the two types of energy (RF and HF).

After each application, the electrode tip will be examined to verify the absence of clot, and cleaned if necessary. Two hours after the procedure, the Triphenyl Tetrazolium Chlorure $2 \%$ would be administered $(1.5 \mathrm{cc} / \mathrm{kg})$, to a better separation between the viable tissue of the necrotic tissue. Then, the animal is then sacrificed; thigh muscles are excised and fixed in formalin $10 \%$. The lesions must be perfectly numbered to be able to measure the maximum depth, the maximum diameter and the diameter of the surface lesion.

\section{Results}

Table 1. Dimensions of lesions obtained by HF and RF methods

\begin{tabular}{|c|c|c|c|c|c|c|}
\hline Thigh 1 & $\begin{array}{l}\text { Great } \\
\text { axis } \\
(\mathrm{mm})\end{array}$ & $\begin{array}{l}\text { Small } \\
\text { axis } \\
(\mathrm{mm})\end{array}$ & $\begin{array}{c}\text { Deep- } \\
\text { ness } \\
(\mathrm{mm}) \\
\end{array}$ & $\begin{array}{c}\text { Type } \\
\text { of } \\
\text { energy }\end{array}$ & $\begin{array}{c}\text { Power } \\
\text { (watts } \\
\text { ) }\end{array}$ & $\begin{array}{l}\text { Dura- } \\
\text { tion (s) }\end{array}$ \\
\hline 1 & 9 & 6 & 4 & RF & 15 & 30 \\
\hline 2 & 10 & 11 & 3 & RF & 9 & 30 \\
\hline 3 & 12 & 8 & 4 & $\mathrm{HF}$ & 10 & 30 \\
\hline 4 & 9 & 7 & 4 & $\mathrm{HF}$ & 30 & 5 \\
\hline 5 & 9 & 7 & 4 & $\mathrm{HF}$ & 30 & 5 \\
\hline \multicolumn{7}{|l|}{ Thigh 2} \\
\hline 1 & 6 & 6 & 3 & RF & 5 & 30 \\
\hline 2 & 8 & 6 & 4 & RF & 11 & 30 \\
\hline $\begin{array}{c}\text { 3(photo } \\
\text { ) }\end{array}$ & 9 & 7 & 5 & RF & 30 & 9 \\
\hline 4 & 9 & 7 & 5 & $\mathrm{HF}$ & 10 & 30 \\
\hline $\begin{array}{c}5 \text { (photo } \\
\text { ) }\end{array}$ & 10 & 9 & 5 & $\mathrm{HF}$ & 30 & 10 \\
\hline 6 & 10 & 7 & 4 & $\mathrm{HF}$ & 50 & 8 \\
\hline
\end{tabular}

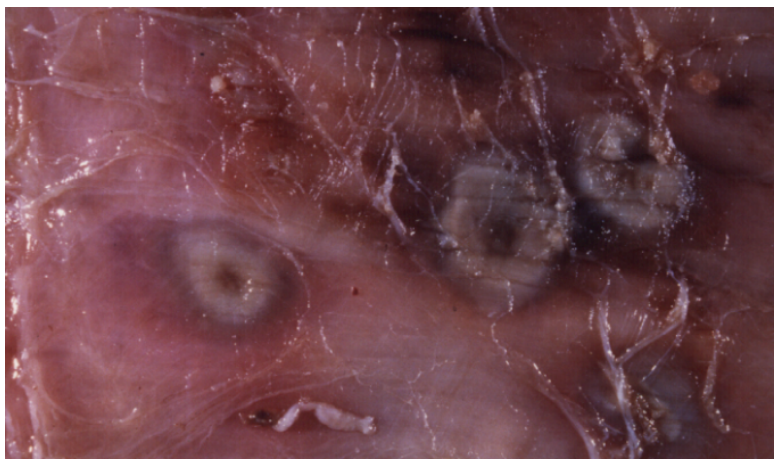

Photo: lesions examples obtained in the same condition a) left injury by RF technique ( $30 \mathrm{w}, 9$ seconds) b) right injury by $\mathrm{HF}$ technique ( $30 \mathrm{w}, 10$ seconds)

Figure 4. The picture shows that the quality of HF lesions confirms that this method fits perfectly in the clinical area 
The table below shows that the lesions obtained by HF method have larger dimensions than the dimensions of the lesions obtained with the RF method.

\section{Discussion}

In sinusoidal case, if we consider a medium without free charges, it appears a current which the first term is the conduction current $\mathrm{J}_{\mathrm{c}}$ and the second a displacement current $\mathrm{J}_{\mathrm{d}}$.

The last subsists only in alternative regime and it propagates without support. At time of ablation (alternative current), probe in contact with the myocardium, transfer its electromagnetic energy directly. So there are two phenomena which cause tissue heating. A first phenomenon concerns the conduction current that causes the collision of ions and whose intensity decreases to $1 / \mathrm{r}^{2}$. The second phenomenon is due to the near field radiation in the proximity of the electrode. It's called displacement current and causes the oscillation and orientation of polar molecules in the direction of the existing field (The biological middle consists mainly of water in large ratio).

The advantage of the HF method is:

It uses the value of the frequency of both types of heating. This provides damage regardless of the orientation of the electrode. This is in the desired direction since currently in clinical ablation; the objective is to obtain lesions regardless of the orientation of the catheter.

Quantity of the radiation heating of the tissue makes the quality of contact electrode-tissue less important. It is also moving towards the obtaining of linear lesions in order to obtain a conduction block. However, RF technique does poorly because we have to make separate points of ablation. HF technique permits easily this kind of lesion which is a very interesting alternative for the future. The use for RF technique in bipolar mode hasn't delivered enough injury wider. The HF technique produces wide enough and deep lesions in bipolar mode compared to RF technique.

We worked to obtain a good correlation between the optimum control of the lesion and the level of the temperature reached by the tissue. But the measurement of temperatures by thermistors or thermocouples is inevitably disrupted by the high frequency signal. The temperature control in HF cannot be done only by using an optical fiber thermometry of very small diameter $(0.8 \mathrm{~mm})$. This optical fiber, unbreakable, will be incorporated within the electrophysiology probes. In practice, we should imagine a temperature measuring exactly halfway between the two electrodes rather than the end. For the bipolar probe, the field is much more intense between the two electrodes.

\section{Conclusions}

It currently lacks a technique that achieves linear lesions; we believe that HF can find its place. Currently, the lines of research using multi-polar catheters using RF technique, but the consistency of the lesions is not perfect as our tests show that the horizontal orientation of the bipolar HF catheter improves the results. These in vivo results need to be reinforced by the almost constant control of temperature during the ablation session. On this plan we are working to formulate a protocol study to confirm the achievement of linear lesions with $\mathrm{HF}$ technique and meetings for intracardiac ablation.

\section{REFERENCES}

[1] S.K. Huang, S. Bharati, A.R. Graham. Closed chest catheter desiccation of the atrioventricular junction using radio frequency energy: a new method of catheter ablation. J. Am. Coll. Cardiol. 18 (1987), 349-358.

[2] T. Lavergne, L.Guize, J.Y. Le Heuzey, Closed-chest atrioventricular junction ablation by high-frequency energy transcatheter desiccation. Lancet; 2 (1986), 858-859.

[3] W.M. Jackman, X.Z. Wang, K.J. Friday, Catheter ablation of accessory atrioventricular pathways (Wolf-Parkinson-White syndrome) by radio frequency current. N. Engl. J. Med; 324 (1991), 1605-1611.

[4] Aymon A.Hussein and al.; Radiofrequency ablation of atrial fibrillation in patients with mechanical mitral valve prosthesis; Journal of the American college of cardiology; Vol. 58, $\mathrm{N}^{\circ} 6$, 2011.

[5] W.M. Jackman, K.J. Beckman, J.H. McClelland, Treatment of supraventricular tachycardia due to atrioventricular nodal re-entry by radio frequency catheter ablation of slow-pathway conduction. N. Engl. J. Med; 327 (1992), 313-318.

[6] S.K.S. Huang; Advances in applications of radiofrequency current to catheter ablation therapy; P.A.C.E., Vol. 14, Junuary 1991.

[7] S.K.S. Huang; Radiofrequency catheter ablation of cardiac arrhythmias: Appraisal of an evolving therapeutic modality; Am.Heart Journal, vol.118, $\mathrm{N}^{\circ} 6,1317-1323,1989$.

[8] S.K. Saksena; Use of radiofrequency energy for catheter ablation of the endomyocardium: A prospective energy source; J. Electrophysiol., 1:78-91, 1987.

[9] Langberg JJ, Chin MC, Rosenqvist $\mathrm{M}$ et al. Catheter ablation of the atrioventricular junction with radio frequency energy; Circulation 1989; 80: 1527-1535.

[10] Hindricks G., Haverkamp W., Rissel U., et al; Experimental observations on the use of radiofrequency energy for ablation of ventricular tissue. New arrhyt. IV(1/2): 337-342, 1988.

[11] J.O. Franklin, J.J. Langberg, O. Michael, W.E. Finkbeiner, J.M. Herre, J.C. Griffin, M. Scheinman; Catheter ablation of canine myocardium with radiofrequency energy; PACE, Vol. 12, Jan. 1989, Part II.

[12] P. Khairy and al.; Enlargement of catheter ablation lesions in infant hearts with cryothermal versus radiofrequency energy: An animal study. Circ. Arrhythm. Electrophydiol; 4; 211-217; 2011.

[13] A. Rouane, D. Kourtiche; Characterization of microwave 
antennas for intracardiac ablation frequencies 915 and 2450 MHz. Measurement Science Review, Vol. 12; № 1, 2012.

[14] A.Rouane, M. Nadi., P.Bru, A. Staiquly, D. Kourtiche, A. Hedjiedj, G. Prieur, Intracardiac HF catheter ablatherapy : Technical aspects. Med. Eng. Phys., 17 (1995), 36-41.

[15] M. Sied; A. Rouane, P.A. Chapelon; M. Nadi; P. Bru, Etude de sondes électrophysiologiques monopolaire et bipolaire en ablathérapie intracardiaque haute fréquence, ITBM, Vol. 18 (1997), $\mathrm{N}^{\circ} 3,219-223$.

[16] P.BRU, P.LAURIBE, A.ROUANE, M.NADI, G.PRIEUR,
P.RICARD, C. DE CHILLOU, E.ALIOT, S.LEVY, "Catheter ablation using very high frequency current: effects on the atrioventricular junction and ventricular myocardium in sheep"; Europace Electrophysiologiy vol 4; 69-75 janvier 2002.

[17] H. Nakagawa, W. S. Yamanashi, Jan V. Pitha, M. Arruda, X. Wang, K. Ohtomo, K. J. Beckman, J. H. McClelland, R. Lazzara, W. M. Jackman ; Comparison of in vivo tissus temperature profile and lesion geometry for radiofrequency ablation with a saline-irrigated electrode versus temperature contrôle in a canine thigh muscle preparation; Circulation, vol. 91, N 8, April 15, 1995. 